\title{
Left on the right: allochiria in a case of left visuo-spatial neglect
}

\author{
Peter W Halligan. John C Marshall, Derick T Wade
}

\begin{abstract}
A patient with left visuo-spatial neglect after right fronto-parietal haemorrhage failed to cross out stimuli in left space on cancellation tests. Her bisections of horizontal lines were displaced significantly to the right of true centre. On drawing and copying tasks, she sometimes omitted features on the left (neglect) and sometimes transposed them to right space (allochiria). This co-occurrence of neglect and allochiria has been observed previously but has provoked little theoretical comment. We draw attention to aspects of the combination that seem critical to the interpretation of visuo-spatial neglect.
\end{abstract}

(F Neurol Neurosurg Psychiatry 1992;55:717-719)

"Hemispatial neglect" is an appropriate description for many of the symptoms displayed by patients with right parietal damage. Consider the patient who fails to cross out target stimuli in contralesional space, ${ }^{1}$ who bisects horizontal lines to the right of centre, ${ }^{2}$ or who copies only the right side of a drawing. ${ }^{3}$ Such cases neglect to process or respond to stimuli in a (variable) portion of visual hemispace. Yet there is (at least) one aspect of the performance of some of these patients for which the term "neglect" does not appear entirely adequate.

For example, in tasks where the patient is required to locate states or cities on a map, targets may be displaced towards the ipsilesional (right) side rather than simply omitted. ${ }^{3}$ A similar phenomenon is found on the familiar "bedside" task of drawing a clockface from memory; some patients transpose all the numbers to the ipsilesional side. ${ }^{4}$ Similarly, on a pointing task, patients may detect a left visual field stimulus but erroneously point to it in the right (ipsilesional) hemispace. ${ }^{5}$ Transpositions can also be observed on purely "imaginal" tasks; when describing a scene from memory (and a specified vantage point) some patients transpose features from the "neglected" to the "good" side."

Phenomena of this general nature (allesthesia or allochiria) were first observed in the tactile modality ${ }^{6}$; a touch on the contralesional side of the body is reported as occurring (often in the symmetrical position) on the ipsilesional side. $^{7}$ Similar referrals from left (affected) to right (normal) space have been reported in audition, ${ }^{8}$ and olfaction. ${ }^{9}$ The classic account of visual (or optic) allochiria-where objects located in one lateral hemispace are perceived in the opposite hemispace-was given by Herrmann and Poetzl. ${ }^{10}$ Meador et al ${ }^{11}$ provide historical analysis of some conceptual and terminological confusions in the area.

It is possible that neglect and allochiria are theoretically-unrelated disorders; their association (or dissociation) in individual patients could be due solely to an anatomical contiguity of discrete modules dedicated to "input" and "output" processes. There is, however, an emerging consensus, ${ }^{51213}$ that the phenomena may be two sides of the same coin. We describe a pertinent example of transposition by a patient who showed left neglect on traditional testing.

\section{Case report}

The patient is a 69 year old, right handed woman who had a right hemisphere stroke on 22 October 1989. This resulted in a severe left sided hemiparesis and a marked loss of tactile sensation on the left (without tactile allochiria or allesthesia). There was neither a visual field deficit nor nystagmus, although extinction to bilateral simultaneous visual confrontation was found. CT scan showed a large fronto-parietal haemorrhage in the right hemisphere (fig 1). On admission to the Rivermead Rehabilitation Centre on 19 February 1990, the outstanding neuropsychological symptom was gross left sided visual neglect. When she was examined on the Behavioural Inattention Test (BIT) ${ }^{14}$ in

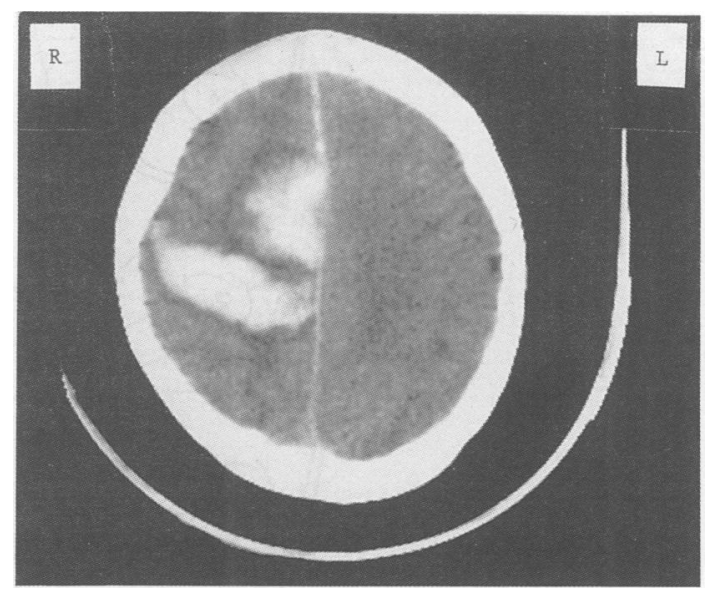

Figure $1 C T$ scan showing a large fronto-parietal haemorrhage in the right hemisphere. Left is on the right of the image. 
May 1990, she scored 73/146. This aggregate is based upon performance on: line crossing, letter- and star-cancellation, figure copying, line bisection and representational drawing. The cut-off score for normal performance is $130 / 146$. On star-cancellation (the most sensitive test from the BIT), the patient failed to cross out any of 27 targets on the left of the stimulus sheet and only succeeded in crossing out $12 / 27$ on the right. Horizontal lines were bisected significantly to the right of centre. On copying simple geometric shapes, features on the left were consistently omitted.

Asked to draw a clockface from memory, the patient's performance was variable. She always drew a complete outline, but on one trial included only the appropriate six numbers on the right, whilst on two trials all twelve numbers were placed along the right hemicircumference. We accordingly devised a new copying task to explore further such transpositions.

\section{Methods}

The stimuli comprised three images of a butterfly, drawn in black ink on white sheets of A4 $(297 \mathrm{~mm} \times 210 \mathrm{~mm})$ paper. The drawings were centred on the stimulus sheet and placed directly in front of the patient's midline. Each
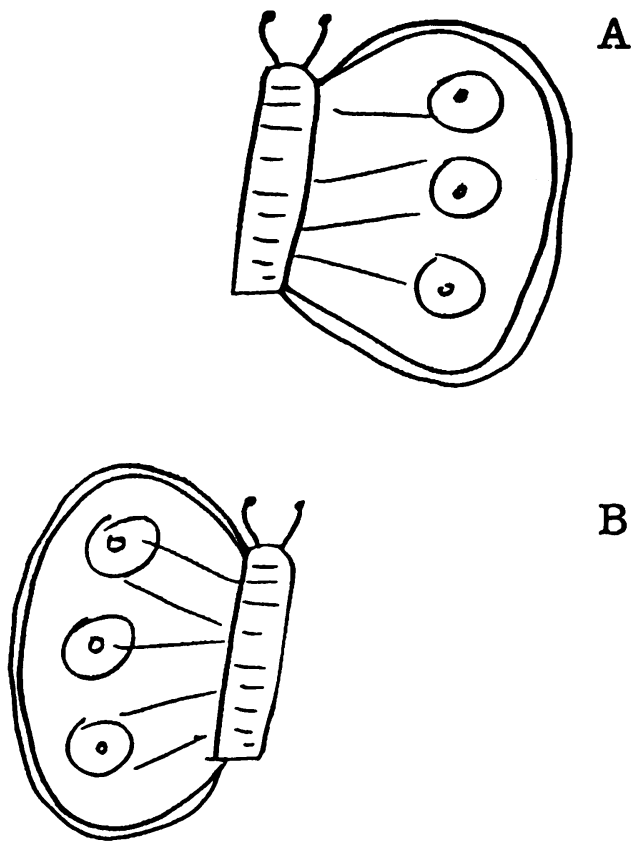

B

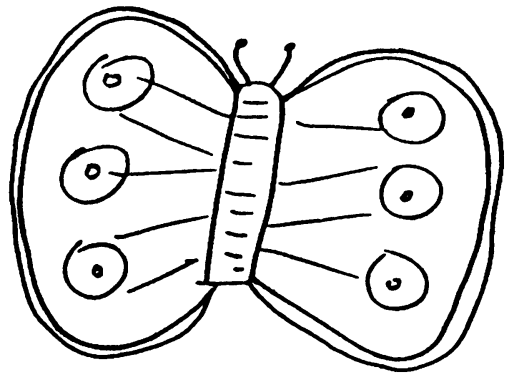

C

Figure 2 Stimuli for copying; butterfly with missing left wing (a), missing right wing (b), and full butterfly (c). drawing was displayed on the desk top immediately above a blank sheet of A4 on which the patient made her copy. The horizontal extent of the complete butterfly (fig 2c) was $194 \mathrm{~mm}$ with a maximal height of $140 \mathrm{~mm}$. The horizontal extent of the right and left butterfly (figs 2a and b) was $115 \mathrm{~mm}$. For all stimuli the body of the butterfly was always centred on the page. No time limit was imposed; the patient inspected the stimulus and her copy until she was satisfied with her performance.

\section{Results}

Stimulus fig 2a shows the butterfly with a missing left wing. The patient reproduced the drawing accurately (fig 3a), omitting only one horizontal marking on the right wing. Stimulus fig $2 \mathrm{~b}$ has a missing right wing. It too is well reproduced (fig $3 b$ ), although with some inaccuracy in the number and orientation of the lines on the wing. The copy of the full butterfly (fig $2 \mathrm{c}$ ) is shown in fig $3 \mathrm{c}$. It clearly shows that details of the left wing have been transposed onto the right wing. The copy shows two sets of markings on the right wing, whereby the internal rings of the left wing of the original have been contrapositioned in "good" space. A second set of three copies produced equivalent results.
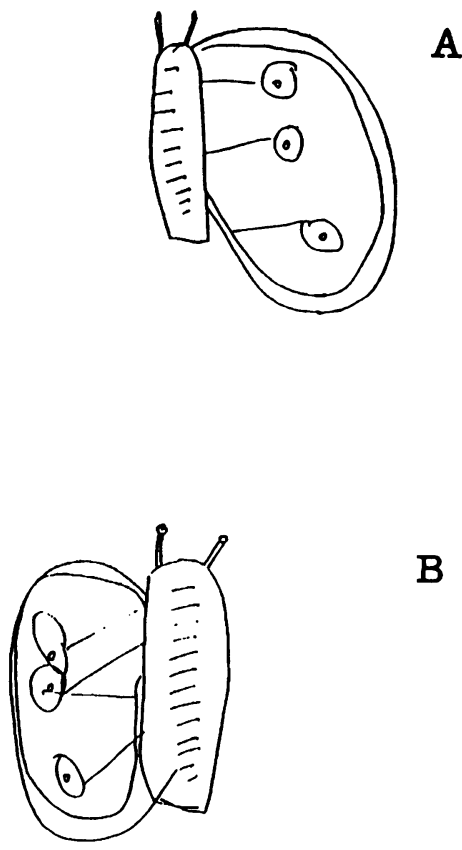

B

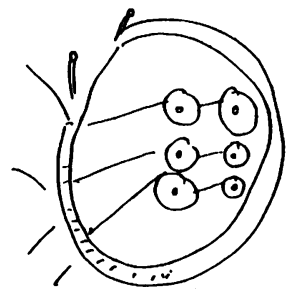

C

Figure 3 The patient's respective copies ( $3 a, b$, and $c)$ of the stimuli $2 a, b$, and $c$. 


\section{Discussion}

It might be argued that "overelaboration" of the right side of a copy may explain some forms of putative transposition. Gainotti and Tiacci $^{15}$ were the first to report that many patients with right hemisphere lesions tend to overscore lines already copied from the right side of the stimulus configuration. A related phenomenon is seen on line cancellation tasks; patients with left neglect may fail to cross out contralesional stimuli whilst making multiple cancellations to lines in ipsilesional space. ${ }^{516}$ This interpretation cannot apply to our present data. When the patient copies the one-winged butterflies, there is no perseveration on any part of the drawing. It is only in the condition where a full butterfly is presented that transposition takes place. Furthermore, the transposition phenomenon is not constant; on cancellation tasks and on copying a symmetrical four-pointed star and flower, the patient made left-sided omissions. In a patient whose tendency to transpose is less variable, it would be pertinent to investigate the effects of stimulus symmetry on allochiria.

Motor hyperkinesia, ${ }^{17}$ whereby the patient is unable or unwilling to execute a motor response to the left of the midsagittal plane, is also ruled out. Despite the fact that the left wing of the butterfly with the right wing missing is situated to the left of the midsagittal plane, the patient's copy (fig $3 \mathrm{~b}$ ) is basically accurate. The sequence of copies ( $3 a, b$ and $c$ ) is reminiscent of visual extinction; both a right wing (3a) and a solitary left wing can be copied (3b). Allochiria appears only when both are simultaneously present (3c).

Visual allochiria raises two problems. First, why do transpositions take place? Second, why do patients apparently fail to notice that their performance in right space is in error? Our patient examined her copy $3 c$ for a considerable time, and on repeated questioning, could detect nothing wrong with it. This latter issue does not apply to auditory, tactile, or olfactory allochiria, where the stimulus is transitory and the response (verbal or manual) is solely indicative of spatial position. The question does apply to all forms of visual allochiria where the response leaves a permanent record on display in right space (a domain in which perception is purportedly normal). When patients inspect the map on which they have placed Los Angeles in Florida why do they not realise that this is geographically bizarre? Also, why do patients who write twelve numbers along the right hemicircumference of a clockface not notice that clocks are not usually so constructed?

All examples of misplacement in neglect indicate that omission and "completion" do not exhaust the expression of the underlying deficit. A more general failure of perceptual parsing must be involved. Los Angeles is west of the east coast of the USA. Does that description satisfy the patient's spatial cognition for map locations and thus cause "neglect" of distance? The numbers on a clock must be ordered from one to twelve. Could the correct sequential order in itself satisfy the patient's sense of "clockness" irrespective of position in space? In figure $3 c$, the butterfly does have two sets of markings, one to the left of the other, and a central body. Is the patient's sense of "normal butterflyness" satisfied by this configuration, and does she not require that in a veridical copy one set of markings should also be left of the body?

The history of art shows how different cultures can adopt different pictorial conventions. The map of the London Underground is perfectly adequate for its intended function; but anyone who attempted to use it on the surface would be seriously misled. Likewise, the conceptual schema of the patient with "neglect" may be satisfied by pictorial objects that represent only a subset of geometric relationships.

This research was supported by the Medical Research Council and the Chest, Heart and Stroke Association.

1 Marshall JC, Halligan PW. Does the midsagittal plane play any privileged role in "left" neglect? Cognitive Neurosychology 1989;6:403-22.

2 Bisiach E, Bulgarelli C, Sterzi R, Vallar G. Line bisection and cognitive plasticity of unilateral neglect of space. Brain and Cognition 1983;2:32-8.

3 Battersby WS, Bender MB, Pollack M, Kahn RL. Unilateral "spatial agnosia" ("inattention") in patients with cerebral lesions. Brain 1956;79:68-93.

4 Bisiach E, Capitani E, Luzzatti C, Perani D. Brain and conscious representation of outside reality. Neuropsychologia 1981;19:543-51.

5 Joanette Y, Brouchon $M$. Visual allesthesia in manual pointing: some evidence for a sensorimotor cerebral organization. Brain and Cognition 1984;3:152-65.

6 Obersteiner $H$. On allochiria: a peculiar sensory disorder. Brain 1882;4:153-63.

7 Kawamura M, Hirayama K, Shinohara Y, Watanabe Y, Sugishita M. Alloaesthesia. Brain 1987;110:225-36.

8 Diamond SP, Bender MB. On auditory extinction and alloacusis. Transactions of the American Neurological Association 1965;90:154-7.

9 Bellas DN, Novelly RA, Eskenazi B, Wasserstein J. The nature of unilateral neglect in the olfactory sensory

system. Neuropsychologia 1988;26:45-52.
10 Herrmann G, Poetzl O. Die optische Alloasthesie. Berlin: Langer, 1928.

11 Meador KJ, Allen ME, Adams RJ, Loring DW. Allochiria vs allesthesia. Is there a misrepresentation? Arch Neurol 1991;48:546-9.

12 Zingerle $\mathrm{H}$. Uber Storungen der Wahrnehmung des eigenen Korpers bei organischen Gehirnerkrankungen. Monatschrift für Psychiatrie und Neurologie 1913;34:13-36.

13 Bisiach E, Berti A. Dyschiria: An attempt at its systematic explanation. In: Jeannerod M, ed. Neurophysiological and Neuropsychological aspects of spatial neglect. Amsterdam: Neuropsychological aspects

14 Halligan PW, Cockburn J, Wilson B. The behavioural assessment of visual neglect. Neuropsychological Rehabilitation 1991;1:5-32.

15 Gainotti C, Tiacci C. Patterns of drawing disability in right and left hemisphere patients. Neuropsychologia 1970, 8:379-84

16 Damasio AR, Damasio H, Chang Chui $H$. Neglect following damage to frontal lobe or basal ganglia. Neuropsychologia 1980;18:123-32.

17 Heilman KM, Bowers D, Coslett HB, Whelan H, Watson RT. Directional hypokinesia: prolonged reaction times for leftward movements in patients with right hemisphere lesions and neglect. Neurology 1985;35:855-9. 